BAG-Rechtsprechung

\title{
DRK-Gesetz: unbefristete Gestellung von Rotkreuzschwestern wird möglich
}

— Das Bundesarbeitsgericht (BAG) hat am 21. Februar 2017 entschieden, dass Rotkreuzschwestern, die bei Gestellungsvertragspartnern, d.h. in Krankenhäusern und anderen Einrichtungen des Gesundheitswesens eingesetzt werden, unter die Regelungen des Arbeitnehmerüberlassungsgesetzes (AÜG) fallen. Auf rund 18.000 der bundesweit 25.000 Rotkreuzschwestern trifft dies zu. „Wir bedauern, dass das BAG nach mehr als 60 Jahren gleichlautender Rechtsprechung seine bisherige Rechtsauffassung nicht vollumfänglich aufrechterhalten hat", sagt Generaloberin Gabriele MüllerStutzer, Präsidentin des Verbandes der Schwesternschaften vom DRK e.V. (VdS).

Auch wenn die Entscheidung des BAG zunächst keine unmittelbaren Auswirkungen auf den Arbeitsplatz von gestellten Rotkreuzschwestern hat, bedeutet die Umsetzung der Vorgaben des AÜG für die DRK-/
BRK-Schwesternschaften einen erheblichen administrativen Mehraufwand (Einholen der Erlaubnis zur Arbeitnehmerüberlassung, namentliche Meldung der gestellten Rotkreuzschwestern an die Agentur für Arbeit etc.).

Der Widerstand des VdS und des DRK richtet sich primär gegen die uneingeschränkte Anwendung des AÜG auf gestellte Rotkreuzschwestern, da sie sich weder formal noch inhaltlich als "Leiharbeitnehmerinnen" verstehen. Das Gros der Rotkreuzschwestern befindet sich in einem unbefristeten Einsatz bei Gestellungsvertragspartnern und gehört zur "Stammbelegschaft" der Gesundheits- und Pflegeeinrichtungen.

Ein wichtiger Kritikpunkt, die Überlassungshöchstdauer von 18 Monaten scheint inzwischen vom Tisch zu sein: Die Bundesregierung und namentlich die Bundesministerin für Arbeit und Soziales, Andrea Nahles, hat

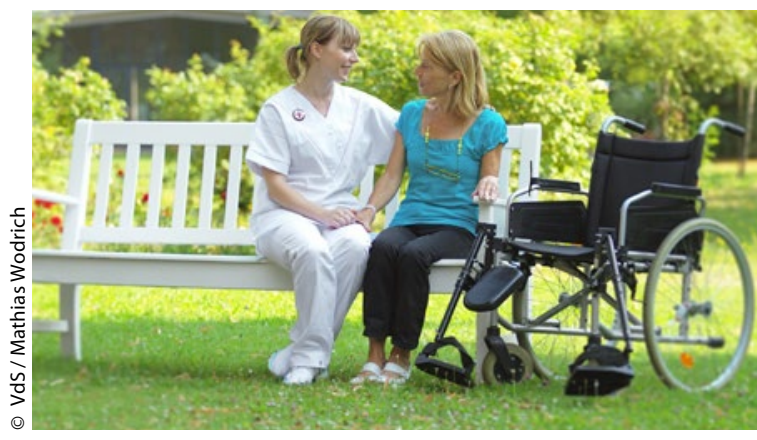

eine zügige Änderung des DRK-Gesetzes zugesagt, der zufolge auch künftig die Gestellung von Mitgliedern einer DRK-Schwesternschaft unbefristet ermöglicht werden kann. Damit wäre sichergestellt, dass Rotkreuzschwestern auch weiterhin dauerhaft an einem Einsatzort tätig sein können.

www.rotkreuzschwestern.de

\section{Hier steht eine Anzeige.}

\section{黑 Springer}

